\title{
Rare Case of Vasculitis of the Hepatic Artery
}

\author{
Padmavathi Mali, MD; Sudheer R. Muduganti, MD; and Jerry Goldberg, MD
}

\begin{abstract}
Vasculitis is an accumulation of inflammatory leucocytes in the blood vessels with reactive damage to mural structures. Isolated vasculitis of the gastrointestinal tract without systemic involvement is rare. We report a unique case of a female patient who presented with abdominal pain, and was found, on serology, to have elevated inflammatory markers without autoantibodies. A computed tomography scan of the abdomen and pelvis was suggestive of vasculitis of the hepatic artery. To the best of our knowledge, this is the first case, to date, of vasculitis of hepatic artery.
\end{abstract}

Keywords: Hepatic artery; Incidental;Vasculitis

$\mathrm{V}$ asculitis is an accumulation of inflammatory leucocytes in the blood vessels with reactive damage to mural structures. Vasculitis of the gastrointestinal tract is a manifestation of systemic vasculitis in $20 \%$ of cases, ${ }^{1}$ but isolated vasculitis of the gastrointestinal tract without systemic involvement is rare. We report a unique case of a female patient who presented with abdominal pain, and was found, on serology, to have elevated inflammatory markers without autoantibodies. A computed tomography scan of the abdomen and pelvis was suggestive of vasculitis of the hepatic artery. To the best of our knowledge, this is the first reported case of vasculitis of the hepatic artery. There have been reports of single organ vasculitis, especially in the intestines, gallbladder, and appendix; however, to date, none have been reported in the liver.

\section{Case Presentation}

A 53-year-old woman with a past history of cholecystectomy was admitted with complaint of epigastric discomfort of 2-days duration. The pain was localized, and she reported bloating and nausea, but denied vomiting, fever, chills, melena, urinary or gastrointestinal complaints.

Physical examination showed the patient was afebrile with stable vital signs. Her cardiovascular and respiratory examinations were normal. Abdominal examination demonstrated minimal tenderness on deep palpation of the epigastrium without distension, guarding, or rigidity. A complete blood count showed normal white blood cell count at $6.3 \times 10^{3} / \mu \mathrm{L}$ and normal hemoglobin at $14.2 \mathrm{~g} / \mathrm{dL}$. Basic metabolic panel and liver enzymes were normal, and amylase and lipase levels were not elevated.

Radiographs of the abdomen revealed normal bowel gas pattern, but a CT scan of the abdomen and pelvis showed circumferential thickening of the wall of the common hepatic artery and its right and left hepatic branches, with a narrowed but patent lumen, suggestive of vasculitis (figure 1). The remaining arteries of the abdomen and pelvis were unremarkable. A CT scan of the abdomen and pelvis taken 2 years prior had been unremarkable. Magnetic resonance angiography (MRA) of the visceral arteries of the abdomen and pelvis confirmed the findings.

The erythrocyte sedimentation rate (ESR) upon admission was elevated at $25 \mathrm{~mm} / \mathrm{hr}$ (normal $0-17 \mathrm{~mm} / \mathrm{hr}$ ) and C-reactive protein (CRP) was elevated at $2.6 \mathrm{mg} / \mathrm{dL}$ (normal $0-1 \mathrm{mg} / \mathrm{dL}$ ). Further history and examination was done to rule out systemic vasculitis in view of the elevated sedimentation rate. The patient denied any new rash, and her examination did not show a rash. She denied arthralgias, and her joint examination was unremarkable. She expressed symptoms of photosensitivity and Raynaud's phenomenon, which had become worse on estrogen therapy.
Corresponding Author:

Dr. Padmavathi Mali

Department of Internal Medicine

Marshfield Clinic

1000 North Oak Avenue

Marshfield,WI 54449

Phone: (7I5) 387-5537

E-mail: mail.padmavthi@marshfieldclinic.org
Received: April 28, 2015

Revised: June 12, 2015

Accepted: June 24, 2015

doi: $10.3121 / \mathrm{cmr} .2015 .1290$ 


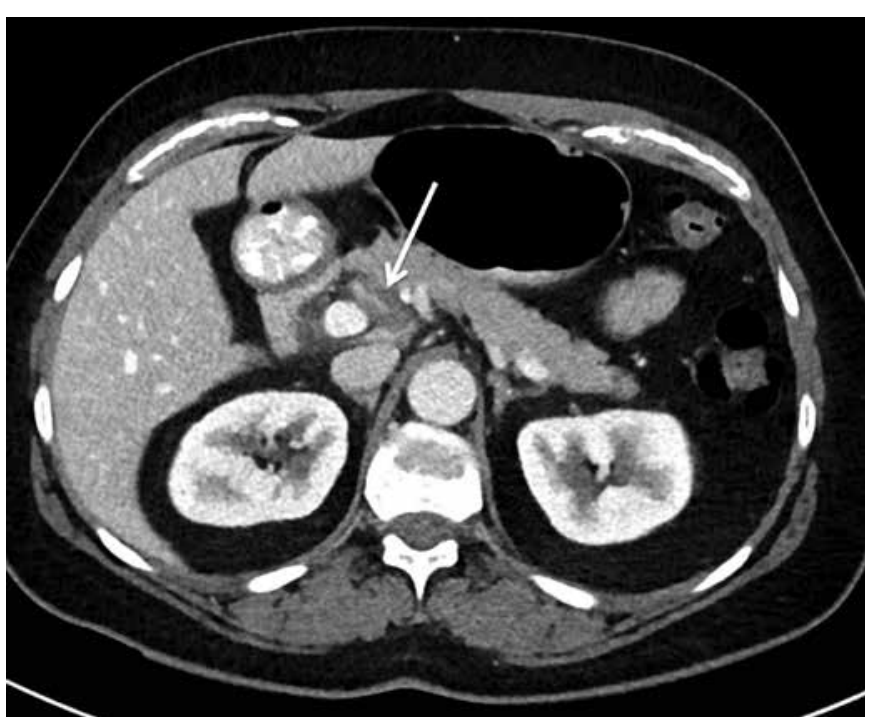

Figure 1: Computed tomography (CT) scan of the abdomen (Axial $3 \mathrm{~mm}$ images with Isovue 300 and oral contrast). The white arrow indicates the common hepatic artery with a thickened rind of soft tissue density and mild stranding in the adjacent fat. The soft tissue density appears circumferential around the narrowed lumen. This is new when compared to a prior CT.

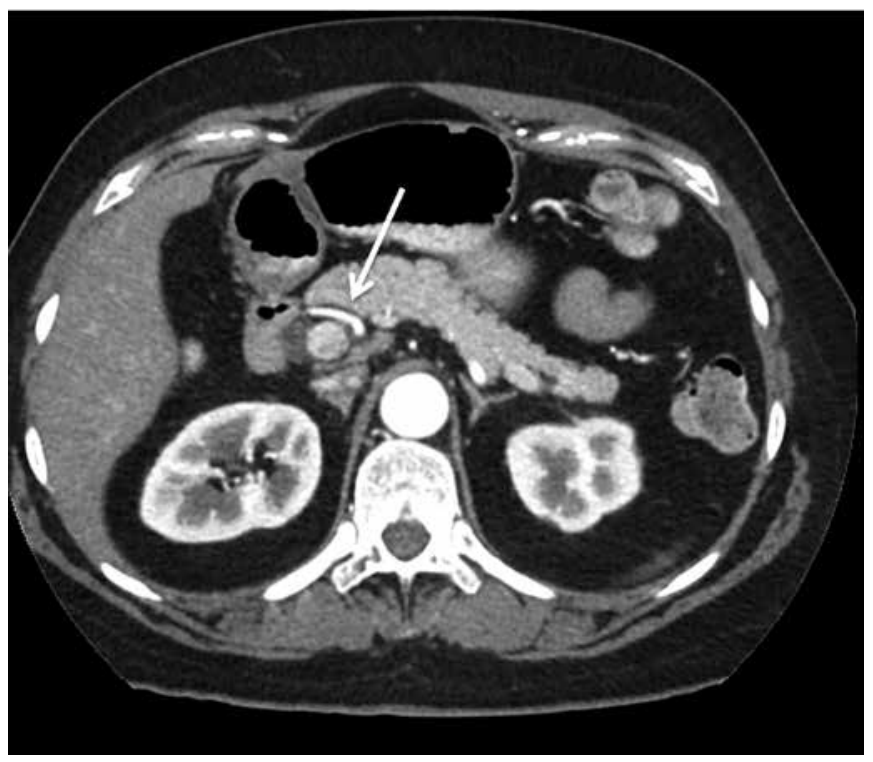

Figure 2: Computed tomography (CT) scan of the abdomen (axial $3 \mathrm{~mm}$ images with Isovue 300 and oral contrast) obtained 12 weeks after the CT shown in Figure 1. The hepatic artery wall thickening is markedly improved, with only minimal residual thickening remaining.

Given the findings on CT scan and MRA with elevated sedimentation rate, evaluation for systemic vasculitis was performed with antinuclear antibody, cytoplasmic antineutrophil cytoplasmic autoantibody, perinuclear neutrophil antibodies, rheumatoid factor, and anti-cyclic citrullinated peptide, all of which were negative. A C4 complement level was normal at $24.6 \mathrm{mg} / \mathrm{dL}$ (range $14-40 \mathrm{mg} / \mathrm{dL}$ ), C3 complement level was normal at $135 \mathrm{mg} / \mathrm{dL}$ (range $83-163 \mathrm{mg} / \mathrm{dL}$ ), and total complement was also normal. Hepatitis B and C, Lyme serology, and cryoglobulin were also all negative.

The patient was started on pulse dose methylprednisone $1000 \mathrm{mg}$ intravenously daily for 3 days, after which her ESR and CRP normalized over the next 2 days, and her symptoms resolved. She was discharged home on prednisone. Prednisone therapy was later tapered, and during the taper, she did not have abdominal pain or gastrointestinal symptoms. Two weeks after prednisone was tapered, a CT scan of the abdomen was done and showed marked improvement in the thickening of the wall, as demonstrated by interval decrease in the soft tissue density surrounding the hepatic artery. The initial CT scan of the abdomen showed thickening of the wall of the hepatic artery and diminished luminal caliber. Several weeks later, the CT scan showed improvement in the thickening (figure 2). At the 6-month follow-up, the patient denied abdominal pain, and erythrocyte sedimentation rate normalized to $12 \mathrm{~mm} / \mathrm{hr}$ (normal 0 -15). Follow-up CT of the abdomen showed significant interval decrease in the soft tissue density surrounding the common hepatic artery and its branches when compared to prior examination.

\section{Discussion}

Vasculitis is defined as accumulation of inflammatory leucocytes in blood vessels with reactive damage to mural structures. Vasculitis should be suspected if a patient presents with systemic symptoms with single or multi-organ dysfunction. This case is unique as the patient presented with abdominal pain and was diagnosed with vasculitis of the hepatic artery. As seen in other cases of gastrointestinal vasculitis, she had elevated inflammatory markers without autoantibodies, as she did not have systemic vasculitis, but had single organ vasculitis.

Definitions for vasculitides adopted by the 2012 International Chapel Hill Consensus Conference on the Nomenclature (CHCC 2012 $)^{2}$ are classified as: large vessel vasculitis as Takayasu Arteritis and giant cell arteritis; medium vessel vasculitis as polyarteritis nodosa and Kawasaki Disease; small vessel vasculitis as anti-neutrophil cytoplasmic antibody associated vasculitis, microscopic polyangiitis, granulomatosis with polyangiitis (Wegener's), eosinophilic granulomatosis with polyangiitis (Churg Strauss), immune complex vasculitis, antiglomerular basement membrane disease, cryoglobulinemic vasculitis, IgA vasculitis (Henoch Schonlein Purpura), and hypocomplementemic urticarial vasculitis (anti C1q vasculitis); and variable vessel vasculitis as Behcet's disease and Cogan's syndrome.

Single organ vasculitis (SOV) is defined as vasculitis in arteries or veins of any size in a single organ that has no features to indicate that it is a limited expression of a systemic vasculitis. Vasculitis distribution may be unifocal or multifocal (diffuse) within an organ. Some patients originally diagnosed as having SOV will develop additional disease manifestations that warrant redefining the case as one of the systemic vasculitides (eg, cutaneous arteritis later becoming systemic polyarteritis nodosa). ${ }^{3}$ 
SOV may affect organs in a diffuse or multifocal fashion (eg, central nervous system and skin) or may be confined to focal sites (eg, breast, gynecologic, testicular, and abdominal structures, and the aorta). Because the territories affected in SOV may also be targeted in systemic vasculitis, the diagnosis of SOV should be applied when it is clear that vascular inflammation is not present in other sites at the time of diagnosis as well as during follow-up surveillance, which is recommended for a period of at least 6 months. Our patient presented clinically with abdominal pain, had elevation of acute phase reactants, and her CT scan was suggestive of vasculitis in the hepatic artery without clinical and laboratory evidence of systemic vasculitis or connective tissue disease. The diagnosis, therefore, was consistent with single organ vasculitis of the liver.

In a case series from the Mayo $\mathrm{Clinic}^{4}$ over a period of 12 years, 18 cases of vasculitis of the gastrointestinal tract were identified, with the esophagus, stomach, small or large bowel, peritoneum, appendix, gallbladder, and pancreas affected. The most common complaint was abdominal pain, and autoimmune workup was negative in all these patients. The diagnosis was established with a combination of symptoms and histopathology suggestive of vasculitis or angiography with high probability findings. Of the patients who had angiography, findings were negative in only one of 18 patients. Treatment was provided in 10 of the 18 patients. Of the 10 patients treated, five received steroids, and the other 5 received both steroids and immunosuppressive medications, with $8 / 10$ responding to treatment. Upon follow-up of the 10 treated patients, $2(20 \%)$ attained remission, $5(30 \%)$ attained relapse, and $3(30 \%)$ died. Of the 8 untreated patients during follow up, $3(37 \%)$ attained remission, $1(12.5 \%)$ had relapse, and $4(50 \%)$ died, indicating higher rates of death in those untreated. After a median follow up of 10 months, none of the 18 patients had evidence of systemic vasculitis. Our patient was treated with steroids with improvement in symptoms and imaging.

Gastrointestinal involvement is a manifestation of systemic vasculitis (polyarteritis nodosa, Henoch-Schonlein purpura, systemic lupus erythematosus, or rheumatoid arthritis) in $20 \%$ of the cases. ${ }^{1,5}$ Single organ vasculitis of the small and large intestines, appendix, gall bladder, stomach, pancreas, esophagus, and omentum have been reported, but not of the liver. Intestines, especially small intestines, are the most commonly involved organs. ${ }^{6} \mathrm{SOV}$ is often found incidentally in the course of biopsies or surgery for suspected malignancy, infection, or structural abnormalities. There have been case reports of acute abdomen and mega-colon resulting from SOV of the intestines. ${ }^{78} \mathrm{SOV}$ of the gallbladder and appendix is usually discovered incidentally during cholecystectomy and appendectomy $y^{9,10}$ and treated with surgical resection; it does not require immunosuppressive medications. SOV involving the pancreas is rare and is managed surgically; it does not need immunosuppressive therapy. ${ }^{1,11}$

The diagnosis always requires exclusion of systemic illness. In focal forms of SOV, certain clinical, laboratory, and pathologic features assist in distinguishing from systemic vasculitis, and consequently, in devising therapeutic and surveillance strategies. ${ }^{3}$ Autoimmune workup is usually negative in these patients, and definitive diagnosis of single organ vasculitis involves exclusion of systemic involvement by thorough history and clinical examination. The ESR is elevated in most patients, and normal ESR makes the diagnosis less likely.

Surgery can be curative in mild cases, but in severe forms, especially for those affecting the small or large bowel, steroid therapy is necessary. In focal SOV, resection of the inflammatory lesion alone may be curative; whereas, systemic therapy is almost always required for diffuse forms of SOV. A major concern is if SOV of the gastrointestinal tract is a localized gastrointestinal vasculitis or an initial manifestation of a more severe systemic vasculitis. Because of this, a complete evaluation to exclude the presence of a potentially threatening systemic vasculitis is required.

\section{Conclusion}

Single organ vasculitis involving the gastrointestinal tract, especially the liver, is very rare. Gastrointestinal vasculitis can be part of systemic vasculitis or isolated vasculitis. If a patient is diagnosed with vasculitis on imaging or by biopsy, careful systemic examination needs to be done, along with autoantibodies to rule out systemic involvement. Patients diagnosed with single organ vasculitis also need close follow up for at least 6 months after diagnosis to look for systemic involvement. Treatment with steroids showing improvement can be done, as in our patient.

\section{Acknowledgements}

The authors thank Dr. Nandita Bhattacharjee of the Marshfield Clinic Radiology/Body Imaging Department for expert assistance in providing and enhancing the images. We also thank the Marshfield Clinic Research Foundation's Office of Scientific Writing and Publication for assistance in preparing this manuscript.

\section{References}

1. Gonzalez-Gay MA, Vazquez-Rodriguez TR, Miranda-Filloy JA, Pazos-Ferro A, Garcia-Rodeja E. Localized vasculitis of the gastrointestinal tract: a case report and literature review. Clin Exp Rheumatol 2008; 26: S101-S104

2. Jennette JC, Falk RJ, Bacon PA, Basu N, Cid MC, Ferrario F, Flores-Suarez LF, Gross WL, Guillevin L, Hagen EC, Hoffman GS, Jayne DR, Kallenberg CG, Lamprecht P, Langford CA, Luqmani RA, Mahr AD, Matteson EL, Merkel PA, Ozen S, Pusey CD, Rasmussen N, Rees AJ, Scott DG, Specks U, Stone JH, Takahashi K, Watts RA. 2012 revised International Chapel Hill Consensus Conference Nomenclature of Vasculitides. Arthritis Rheum 2013;65:1-11.

3. Hernández-Rodríguez, José Hoffman, Gary S. Updating singleorgan vasculitis. Curr Opin Rheumatol 2012;24:38-45.

4. Salavarani C, Calamia KT, Crowson CS, Miller DV, Boradwell AW, Hunder GG, Matteson EL, Warrington KJ. Localized vasculitis of the gastrointestinal tract: a case series. Rheumatology 2010;49:1326-1335.

5. Mulder AH, Horst G, Haagsma EB, Limburg PC, Kleibeuker JH, 
Kallenberg CG. Prevalence and characterization of neutrophil cytoplasmic antibodies in autoimmune liver diseases.

Hepatology 1993;17:411-417.

6. Müller-Ladner U. Vasculitides of the gastrointestinal tract. Best Pract Res Clin Gastroenterol 2001;15:59-82.

7. Bisceglia M, Germani G, Tardio B, Di Mattia A, Li Bergoli M. Leukocytoclastic vasculitis of the colon. Ital J Surg Sci 1989;19: 269-272.

8. Vlahos K, THeodoropoulos GE, Lazaris AC, Tahteris E, Panoussopoulos D. Isolated colonic leukocytoclastic vasculitis causing segmental megacolon: report of a rare case. Dis Colon Rectum 2005;48:167-171.

9. Kumar B, Krishnani N, Misra R, Pandey R. Isolated necrotizing vasculitis of gallbladder: a reportof two cases and review of literature. Indian J Pathol Microbiol 2003;46:429-431.

10. Plaut A. Asymptomatic focal arteritis of the appendix; 88 cases. Am J Pathol 1951;27:247-263.

11. Burke AP, Sobin LH, Virmani R. Localized vasculitis of the gastrointestinal tract. Am J Surg Pathol 1995;19: 338-349.

\section{Author Affiliations}

Padmavathi Mali, MD*; Sudheer R. Muduganti, MD* and Jerry Goldberg, MD†

*Department of Internal Medicine, Marshfield Clinic, Marshfield, Wisconsin USA

†Department of Rheumatology, Marshfield Clinic, Marshfield, Wisconsin USA 